correct decay rates for $D=0$, because any single point moment at time $t$ depends on all the moments at $t=0$.

The distribution function approach obviously preserves (2). Let $P^{t}[\Psi \mid \boldsymbol{u}]$ denote the conditional probability functional for the concentration field $\psi(\mathbf{x})$, given the velocity field $\mathfrak{u}(\mathbf{x})$. Consider the $\mathbf{x}$ space discrete so that elemental volume of $\Psi$ space exists. The method of Edwards ${ }^{7}$ gives the following continuity equation:

$$
\begin{aligned}
& \frac{\partial}{\partial t} P^{t}[\Psi \mid \boldsymbol{u}]=\int d^{3} x \frac{\delta}{\delta \psi(\mathbf{x})} \\
& \cdot\left[C \psi^{2}(\mathbf{x})+\mathbf{u}(\mathbf{x}) \cdot \nabla \psi(\mathbf{x})-D \nabla^{2} \psi(\mathbf{x})\right] P^{t}[\Psi \mid \boldsymbol{u}],
\end{aligned}
$$

where $\delta / \delta$ denotes functional differentiation. The single point probability density function is obtained by multiplying (5) by $F(v)$, the probability density functional of $\mathrm{U}$ for all $\mathbf{x}$ and $t$, and integrating over all $\boldsymbol{v}$ and over $\Psi$ for all points but $\mathbf{x}$, resulting in

$$
\begin{aligned}
\left(\frac{\partial}{\partial t}-\right. & \left.C \frac{\partial}{\partial \psi} \psi^{2}\right) P_{x}^{t}(\psi) \\
= & -D \lim _{\mathbf{x}^{\prime} \rightarrow \mathbf{x}} \frac{\partial}{\partial \psi} \nabla_{x^{\prime}}^{2} \int d \psi^{\prime} \psi^{\prime} P_{x, x^{\prime}}^{t}\left(\psi, \psi^{\prime}\right) \\
& -\int d^{3} u \mathbf{u} \cdot \nabla P_{x, x}^{t}(\psi, \mathfrak{u})
\end{aligned}
$$

which reduces to (2) if $D=0$ and if $\Gamma$ and $U$ are jointly homogeneous. An unclosed hierarchy of equations for the multipoint probability density functions can be set up analogous to that for the ordinary turbulence problem, ${ }^{8,9}$ the next member being

$$
\begin{aligned}
{\left[\frac{\partial}{\partial t}-C\left(\frac{\partial}{\partial \psi} \psi^{2}+\frac{\partial}{\partial \psi^{\prime}} \psi^{\prime 2}\right)\right] P_{x, x^{\prime}}^{t}\left(\psi, \psi^{\prime}\right) } \\
=-D\left\{\lim _{\mathbf{x}^{\prime \prime} \rightarrow \mathbf{x}} \frac{\partial}{\partial \psi}+\lim _{\mathbf{x}^{\prime \prime} \rightarrow \mathbf{x}^{\prime}} \frac{\partial}{\partial \psi^{\prime}}\right\} \nabla_{x^{\prime \prime}}^{2} \\
\cdot \int d \psi^{\prime \prime} \psi^{\prime \prime} P_{x, x^{\prime}, x^{\prime}}^{t}\left(\psi, \psi^{\prime}, \psi^{\prime \prime}\right) \\
-\int d^{3} u \mathbf{u} \cdot\left[\nabla_{x} P_{x, \mathbf{x}^{\prime}, x}^{t}\left(\psi, \psi^{\prime}, \mathbf{u}\right)\right. \\
\left.+\nabla_{x^{\prime}} P_{x, x^{\prime}, x^{\prime}}^{t}\left(\psi, \psi^{\prime}, \mathbf{u}\right)\right] .
\end{aligned}
$$

The velocity terms may be treated by considering the parallel hierarchy of joint probability density functions $P_{x, x}^{t}(\psi, u)$, etc., also obtained from (5) without use of the Navier-Stokes equations and formally similar to Eqs. (6) but without a $\mathbf{u}$ integration. Closure may be obtained by parameterization of assumed higher order joint probability density functions.

Let us turn our attention to the reciprocal of the concentration of the contaminant, its specific volume $\Upsilon(\mathbf{x}, t) \equiv \Gamma^{-1}(\mathbf{x}, t)$ (use $\Upsilon^{N-1}$ for $N$ th order reactions), whose dynamical equation is

$$
\Re(\mathbf{x}, t) \Upsilon(\mathbf{x}, t)=C-\frac{2 D}{\Upsilon(\mathbf{x}, t)}|\nabla \Upsilon(\mathbf{x}, t)|^{2} .
$$

The logarithmic nonlinearity in $(7)$ is present in single point hierarchy equations for both (1) and (7),

$$
\begin{aligned}
& \Re \Gamma^{n}=-n C \Gamma^{n+1}-(n-1) D(\nabla \log \Gamma) \cdot\left(\nabla \Gamma^{n}\right), \\
& \Re \Upsilon^{n}=n C \Upsilon^{n-1}-(n+1) D(\nabla \log \Upsilon) \cdot\left(\nabla \Upsilon^{n}\right) .
\end{aligned}
$$

The dissipative term is usually avoided by considering the $\Gamma$ hierarchy of multiple point correlations. After closure certain points are made to coincide before substitution into lower order equations. Unfortunately, the log term appears in the first equation of the $\Upsilon$ hierarchy and cannot be avoided. As a rough approximation one could replace $\Upsilon^{-1}$ by $\langle\Upsilon\rangle^{-1}$ and use a moment closure. Because the dissipative term disappears when $D=0$, O'Brien's invariance is automatically satisfied. Furthermore, when the turbulence is also homogeneous the hierarchy of $\left\langle\Upsilon^{n}\right\rangle$ equations is closed at each level, giving the exact result $d\langle\Upsilon\rangle / d t=C$ and all the central moments of $\Upsilon$ constant in time.

The author is grateful for many beneficial consultations with Dr. J. R. Herring.

* National Research Council-National Aeronautics and Space Administration Resident Research Associate, now at Shell Development Company, Emeryville, California.

1 E. E. O'Brien, Phys. Fluids 9, 1561 (1966).

2 E. E. O'Brien, Phys. Fluids 11, 2087 (1968)

${ }^{3} \mathrm{~S}$. Corrsin, Phys. Fluids 1, 42 (1958).

4 For further details of this note see J. C. Hill, Goddard Space Flight Center Document X-641-69-108 (1969).

5 See also Eq. (42) of S. Corrsin, in Mecanique de la Turbu-

lence, A. Favre, Ed. (Centre National de la Recherche Scientifique, Paris, 1962), p. 27.

6 E. E. O'Brien, Phys. Fluids 11, 2328 (1968).

' S. F. Edwards, J. Fluid Mech. 18, 239 (1964)

8 T. S. Lundgren, Phys. Fluids 10, 969 (1967).

9 A. S. Monin, Prikl. Mat. Mekh. 31, 1057 (1967) [J. Appl. Math. Mech. 31, 1057 (1967)].

\section{Direct Correlation Function in Space and Time}

\author{
F. Lino \\ Department of Physics, North Carolina State University \\ Raleigh, North Carolina \\ (Received 29 August 1969)
}

A new, simpler derivation is presented of the equation suggested by Percus and Yevick to define the generalized direct correlation function in space and time. In addition, an explicit, though formal, definition of this function is given.

The time-dependent generalization of the total correlation function $G(\mathbf{r}) \equiv g(\mathbf{r})-1$ used in the 
classical equilibrium theory of fluids is the densitydensity correlation function"

$$
\hat{G}(\mathbf{r}, t) \equiv(n N)^{-1} \int d \mathbf{r}^{\prime}\left\langle R\left(\mathbf{r}^{\prime}, 0\right) R\left(\mathbf{r}^{\prime}+\mathbf{r}, t\right)\right\rangle,
$$

where

$$
\begin{aligned}
& R(\mathbf{r}, t)=\exp (-t \mathfrak{L}) R(\mathbf{r}, 0), \\
& R(\mathbf{r}, 0)=\sum_{i=1}^{N} \delta\left(\mathbf{r}-\mathbf{r}_{i}\right)-n,
\end{aligned}
$$

and where $\mathscr{L}$ is the Liouville operator (i.e., $\mathscr{L} F$ is the Poisson bracket of the Hamiltonian $H$ with $F), N$ is the number of particles in the volume $V$, and $n$ is the mean density. The brackets denote an equilibrium ensemble average. The initial value of $\hat{G}(\mathbf{r}, t)$ is then, from (1),

$$
\hat{G}(\mathbf{r}, 0)=G(\mathbf{r})+n^{-1} \delta(\mathbf{r}) .
$$

Recently, Percus and Yevick ${ }^{2}$ have suggested a defining equation for the time-dependent extension $\hat{C}(r, t)$ of the equilibrium direct correlation function $C(\mathbf{r})$, to wit,

$$
\begin{aligned}
\frac{\partial \hat{G}(\mathbf{r}, t)}{\partial t}=- & \frac{n}{m \beta} \nabla^{2} \int_{0}^{t} d t^{\prime} \\
& \cdot \int d \mathbf{r}^{\prime} \hat{C}\left(\mathbf{r}^{\prime}, t^{\prime}\right) \hat{G}\left(\mathbf{r}-\mathbf{r}^{\prime}, t-t^{\prime}\right),
\end{aligned}
$$

where $m$ is the mass of a particle and $\beta$ is $\left(k_{B} T\right)^{-1}$ and where initially

$$
\widehat{C}(\mathbf{r}, 0)=C(\mathbf{r})-n^{-1} \delta(\mathbf{r}) .
$$

It is the purpose of this note to present a simpler derivation of Eqs. (5) and (6) than that given in Ref. 2 and in the process to obtain an explicit, though formal, expression for the function $\hat{C}(\mathbf{r}, t)$.

The derivation is a straightforward application of Zwanzig's ${ }^{3}$ projection operator technique to the Fourier transform of Eq. (1),

$$
\hat{G}_{\mathbf{k}}(t)=(n N)^{-1}\left\langle R_{-\mathbf{k}}(0) R_{\mathbf{k}}(t)\right\rangle .
$$

[We write the transform $F_{\mathrm{k}}$ of any function $F(\mathbf{r})$ defined in $V$ such that

$$
\begin{aligned}
F(\mathbf{r}) & =(2 \pi)^{-3} \int d \mathbf{k} F_{\mathbf{k}} \exp (i \mathbf{k} \cdot \mathbf{r}) \\
F_{\mathbf{k}} & =\int d \mathbf{r} F(\mathbf{r}) \exp (-\imath \mathbf{k} \cdot \mathbf{r})
\end{aligned}
$$

for infinitely large $V$.] To evaluate the average in Eq. (7) we do not need to know the general time behavior of $R_{\mathrm{k}}(t)$ but rather only that of its projection onto the "fixed state" $R_{\mathrm{k}}(0)$. To this end, we define, following $Z$ wanzig, a projection operator $P$ such that for any phase-dependent quantity $Q$

$$
\odot Q \equiv \frac{\left\langle R_{-\mathbf{k}}(0) Q\right\rangle}{\left\langle R_{-\mathbf{k}}(0) R_{\mathbf{k}}(0)\right\rangle} R_{\mathbf{k}}(0) .
$$

Note that we may then write

$$
\hat{G}_{\mathrm{k}}(t)=(n N)^{-1}\left\langle R_{-\mathrm{k}}(0) \propto R_{\mathrm{k}}(t)\right\rangle,
$$

and so

$$
\partial \hat{G}_{\mathbf{k}}(t) / \partial t=(n N)^{-1}\left\langle R_{-\mathbf{k}}(0) \partial \rho R_{\mathbf{k}}(t) / \partial t\right\rangle .
$$

The equation of motion of the complete $R_{\mathrm{k}}(t)$,

$$
\partial R_{\mathbf{k}}(t) / \partial t=-\& R_{\mathbf{k}}(t)
$$

is obtained immediately from Eq. (2). To find the corresponding equation for just the projected part $\odot R_{\mathrm{k}}(t)$ we operate on Eq. (12) with $\rho$ and with $(1-\odot)$, obtaining two equations linking the projected part and the "remainder" $(1-P) R_{\mathrm{k}}(t)$. The "remainder" is eliminated, yielding a closed, formal equation for $\rho R_{\mathrm{L}}(t)$; we refer to $Z_{\text {wanzig }}{ }^{3}$ for the details of this calculation. The result is

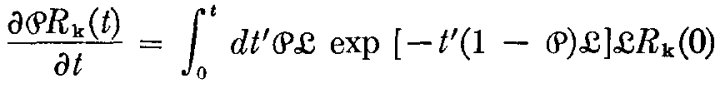

$$
\begin{aligned}
& \frac{\left\langle R_{-\mathbf{k}}(0) R_{\mathbf{k}}\left(t-t^{\prime}\right)\right\rangle}{\left\langle R_{-\mathbf{k}}(0) R_{\mathbf{k}}(0)\right\rangle},
\end{aligned}
$$

so that, with Eq. (11), we have

$$
\begin{aligned}
\frac{\partial G_{\mathrm{k}}(t)}{\partial t}= & \int_{0}^{t} d t^{\prime} \frac{\left\langle R_{-\mathrm{k}}(0) \mathcal{L} \exp \left[-t^{\prime}(1-\mathcal{P}) \mathscr{L}\right] \& R_{\mathrm{k}}(0)\right\rangle}{\left\langle R_{-\mathrm{k}}(0) R_{\mathbf{k}}(0)\right\rangle} \\
& \cdot \hat{G}_{\mathbf{k}}\left(t-t^{\prime}\right) \\
= & -\int_{0}^{t} d t^{\prime} K_{\mathrm{k}}\left(t^{\prime}\right) \hat{G}_{\mathrm{k}}\left(t-t^{\prime}\right)
\end{aligned}
$$

where we have put

$K_{\mathrm{k}}(t) \equiv \frac{\left\langle\mathscr{L} R_{-\mathrm{k}}(0) \exp [-t(1-\mathcal{P}) \mathcal{L}] \mathscr{L} R_{\mathrm{k}}(0)\right\rangle}{\left\langle R_{-\mathrm{k}}(0) R_{\mathrm{k}}(0)\right\rangle}$.

The initial value of the kernel $K_{\mathrm{k}}(t)$ may easily be evaluated

$$
K_{\mathrm{k}}(0)=\frac{k^{2}}{m \beta n \hat{G}_{\mathbf{k}}(0)} .
$$

But, in the equilibrium theory of fluids the direct correlation function is defined by

$$
\left(1+n G_{\mathrm{k}}\right)^{-1} \equiv\left[n \hat{G}_{\mathrm{k}}(0)\right]^{-1}=1-n C_{\mathrm{k}},
$$

so that (16) may also be written

$$
K_{\mathrm{k}}(0)=-\frac{n k^{2}}{m \beta}\left(C_{\mathrm{k}}-\frac{1}{n}\right) .
$$

In general, we define a function $\hat{C}_{\mathbf{k}}(t)$ such that

$$
K_{\mathbf{k}}(t)=-\frac{n k^{2}}{m \beta} \hat{C}_{\mathbf{k}}(t)
$$


Then (14) becomes

$$
\frac{\partial \hat{G}_{\mathbf{k}}(t)}{\partial t}=\frac{n k^{2}}{m \beta} \int_{0}^{t} d t^{\prime} \hat{C}_{\mathbf{k}}\left(t^{\prime}\right) \hat{G}_{\mathrm{k}}\left(t-t^{\prime}\right)
$$

the inverse transform of which is just Eq. (5). Thus, we have verified Percus and Yevick's suggestion that Eq. (5) can serve to define a timedependent direct correlation function which reduces to the equilibrium function at the initial time. In addition, we are able to give the explicit definition of this function, namely,

$$
\begin{aligned}
& \hat{C}_{\mathbf{k}}(t) \equiv-\frac{1}{n^{2} C_{\mathrm{k}}(0)}
\end{aligned}
$$

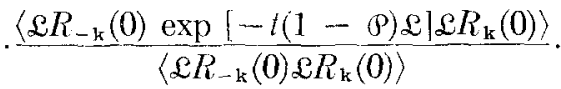

And finally, it is evident that this result is but a special case of the general formalism for time correlation functions developed by Zwanzig. ${ }^{3}$

This work was supported by the North Carolina Engineering Foundation.

${ }^{1}$ L. van Hove, Phys. Rev. 95, 249 (1954).

2 J. K. Percus and G. J. Yevick, Phys. Fluids 11, 2050 (1968).

${ }^{3} \mathrm{R}$. Zwanzig, in Lectures in Theoretical Physics, W. E. Brittin, W. B. Downs, and J. Downs, Eds. (Interscience Publishers, Inc., New York, 1961) Vol. 3, p. 135.

\section{Blast Waves and Scaling Laws}

\author{
Donald L. Jones \\ General Electric Company, Philadelphia, Pennsylvania \\ (Received 11 August 1969; final manuscript received \\ 3 December 1969)
}

Spherical blast wave scaling is studied with the intermediate strength formalism. Simple scaling relations rarely exist, but the intermediate strength equations permit a direct comparison of arrival times and front overpressures for different explosions.

In the evaluation of the effects of explosions under one set of conditions, it is often convenient to compare them with the measured effects of a know explosion under different conditions. The need to compare, rather than compute, explosion effects arises from the complexity of the fluid equations of motion, which are nonlinear. Only for the case of strong blast waves $\left(\Delta P / P_{0}>10\right)$ is there an analytical, closed form solution. ${ }^{1}$ Since the region $\Delta P / P_{0}<10$ is quite important (some shock damage occurs for overpressures as low as $1 \mathrm{psi}$ ), and since the shock spends over $95 \%$ of its lifetime in the overpressure region between 10 atm and 1 psi, a scaling approach developed by Sachs $^{2}$ has been widely used. Although Sachs scaling is a general procedure, its principal application has been in relating the distance from the origin for the occurrence of a given overpressure ${ }^{3}$

$$
\frac{D}{D_{1}}=\left(\frac{W}{W_{1}}\right)^{1 / 3}
$$

where $D$ is the distance from the origin to the shock front and $W$ is the energy. Other important relationships are the variation of the overpressure at a particular location or the arrival time ratio. Unfortunately, such relations are not easily found with scaling relations.

It is the purpose of this note to set down explicitly certain "scaling" relations derived from the intermediate strength blast relations. ${ }^{4}$ For brevity we limit this discussion to spherical blasts, although both the cylindrical and plane cases could be handled in a similar manner.

From Jones ${ }^{4}$ we write the equation for the trajectory of the shock front for a spherical blast

$$
\tau=0.543\left[\left(1+4.61 X^{5 / 2}\right)^{2 / 5}-1\right],
$$

and the overpressure at the front is

$\frac{\Delta P}{P_{0}}=\frac{2 \gamma}{\gamma+1} \frac{4}{25}\left\{0.611\left[\left(1+5.89 X^{3}\right)^{5 / 18}-1\right]\right\}^{-1}$,

where $\tau=a_{0} t / R_{0}, X=D / R_{0}, a_{0}$ is the speed of sound in the undisturbed medium, $R_{0}$ is the characteristic radius $\left[\frac{25}{4}(1 / B)(1 / \gamma)\left(W / P_{0}\right)\right]^{1 / 3}, D$ is the distance from the shock front to the source, $B$ is a constant, ${ }^{5} \gamma$ is the specific heat ratio, $P_{0}$ is the ambient pressure, and $W$ is the energy input to the shock from the explosion. For strong blast waves $\tau=X^{5 / 2}$ and $\Delta P / P_{0}=[2 \gamma /(\gamma+1)]_{\frac{4}{25}} X^{-3}$.

As an example, we derive the scaling relation for the distance at which a given overpressure will occur, assuming different energy releases. Utilizing (3) and some algebra we find $\Delta P / \Delta P_{1}=$ $X_{1}^{3} / X^{3}$. But $\Delta P=\Delta P_{1} ; X_{1}=X$. From the definition of $X$, we now write $D / D_{1}=R_{0} / R_{01}$. This is equivalent to $D / D_{1}=\left(W / W_{1}\right)^{1 / 3}$, which is in accord with Ref. 2 [Eq. (3.56.1)]. Following the same procedure leads to the relations shown in Table I. In addition to the relationship between the given variables and the intermediate formalism, the limiting cases of strong and weak shocks (columns 4 and 5) are provided for comparison.

From Table I, it is clear that only relation (a) is constant for all shocks. The remaining situations have scaling factors that depend on the shock strength; however, Eqs. (2) and (3) provide a means of comparing shock front effects throughout the range of overpressures to as low as 1 psi.

1 G. I. Taylor, Proc. Roy. Soc. (London) A201, 159 (1950).

${ }^{2}$ U. Ericsson and K. Edin, Phys. Fluids 3, 893 (1960).

${ }^{3} \mathrm{~S}$. Glasstone, Ed., The Effects of Nuclear Weapons (U. S. 